\title{
Paraganglioma presenting as a mesenteric cystic mass: A case report
}

\author{
ANDREEA ILIESIU $^{1,2}$, IRENA ANTONIA UNGUREANU ${ }^{1}$, AIDA PETCA $^{3,4}$, \\ MARIA MAGDALENA CONSTANTIN ${ }^{3,5}$, RAZVAN-COSMIN PETCA ${ }^{3,6}$, FLORICA SANDRU ${ }^{3,7}$, \\ TRAIAN CONSTANTIN $^{6}$ and MIHAI CRISTIAN DUMITRASCU ${ }^{3,8}$
}

\author{
${ }^{1}$ Department of Pathology, University and Emergency Hospital, 014461 Bucharest; ${ }^{2}$ Department 2 - Preclinical Sciences, \\ and ${ }^{3}$ Department 3 - Clinical Sciences, 'Carol Davila' University of Medicine and Pharmacy, 050474 Bucharest; \\ ${ }^{4}$ Department of Obstetrics and Gynecology, 'Elias' Emergency University Hospital, 011461 Bucharest; \\ ${ }^{5}$ Department of Dermatology II, Colentina Clinical Hospital, 020125 Bucharest; ${ }^{6}$ Department of Urology, \\ 'Prof. Dr. Theodor Burghele' Clinical Hospital, 050653 Bucharest; ${ }^{7}$ Department of Dermatology, \\ 'Elias' University Emergency Hospital; ${ }^{8}$ Department of Obstetrics and Gynecology, \\ University and Emergency Hospital, 014461 Bucharest, Romania
}

Received January 10, 2020; Accepted February 12, 2020

DOI: $10.3892 / \mathrm{etm} .2020 .8805$

\begin{abstract}
The present study aims to raise awareness about the necessity of a thorough differential diagnosis process when diagnosing paraganglioma as it is an extremely rare entity with a high malignant potential. A 64-year-old female patient was admitted to the hospital with symptoms of bowel obstruction and a palpable abdominal mass. Abdominal and pelvic computed tomography revealed a cystic heterogeneously enhanced mass. The tumor was surgically removed and then microscopically analysed. Microscopically, the mass was composed of nests of small polygonal and round cells with central vesicular nuclei, consistent with the diagnosis of mesenteric paraganglioma which was later confirmed by immunohistochemistry. The critical markers for paragangliomas, namely chromogranin A and $\mathrm{S} 100$, should be used as the first step in diagnosis, followed by other valuable immunohistochemical markers. A long-term follow-up is extremely important following the diagnosis of paraganglioma as all these tumors have a malignant potential.
\end{abstract}

Correspondence to: Dr Florica Sandru, Department of Dermatology, 'Elias' University Emergency Hospital, 17 Marasti Boulevard, 014461 Bucharest, Romania

E-mail: florysandru@yahoo.com

Abbreviations: GFAP, glial fibrillary acidic protein; NETs, neuroendocrine tumors; Ki-67, antigen Ki-67; AE1/AE3, pan cytokeratin; CD117, clusted of differentiation 117; HMB45, melanoma marker antibody; GAPP, adrenal pheochromocytoma and paraganglioma; WHO, World Health Organization

Key words: paraganglioma, neuroendocrine tumor, differential diagnosis, histological features

\section{Introduction}

Tumors deriving from chromaffin cells are rare entities and can be divided into pheochromocytomas and paragangliomas. Paraganglioma is a neuroendocrine tumor (NET) originating in the paraganglia of the sympathetic and parasympathetic nervous system. Paraganglia exhibit a widespread distribution in the body, therefore paragangliomas can occur nearly anywhere except within the bone and the brain (1). Mesenteric origin of these tumors is highly unlikely and only a few cases have been reported in the literature $(2,3)$. We present a challenging case of an unusual cystic tumor of the mesentery proved to be a paraganglioma.

\section{Case report}

A 64-year-old female patient was admitted to the hospital with symptoms of bowel obstruction and a palpable abdominal mass. The patient was living in an urban environment and did not have a significant prior medical history. Abdominal and pelvic computed tomography revealed a cystic heterogeneously enhanced mass measuring $80 / 66 \mathrm{~mm}$ in close proximity to the right fallopian tubes, uterus and the small intestine. Furthermore, left adrenal hyperplasia was observed on the computed tomography.

The tumor and the surrounding mesentery were surgically removed as well as a segment of the corresponding small intestine. Grossly, the tumor was located exclusively inside the mesentery and it showed lesions of cystic degeneration and hemorrhage (Fig. 1).

The histopathological examination was performed on paraffin-embedded tissue samples which were further stained with classical hematoxylin and eosin staining process.

\section{Results}

Microscopically, the mass was composed of nests of small polygonal and round cells with central vesicular nuclei. 


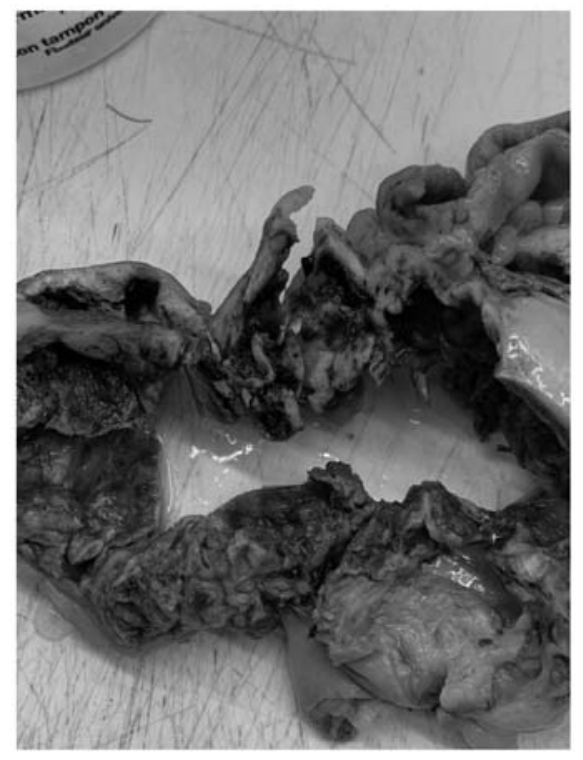

Figure 1. Macroscopic aspect of the surgical specimen. The cystic wall of the tumor and areas of hemorrhage can be observed.

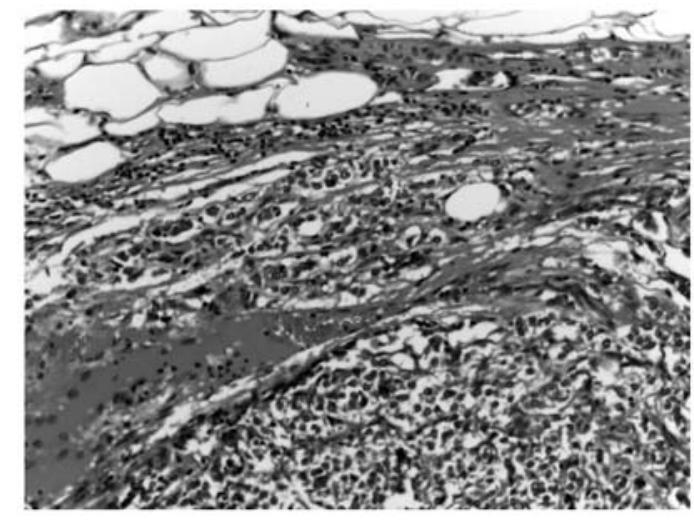

Figure 2. Invasion of tumor cells in the capsule and surrounding adipose tissue (hematoxylin and eosin, $\mathrm{x} 20$ ).

Some of them showed either eosinophilic or clear cytoplasm. The nests were separated by connective tissue septa and the capsule of the tumor was infiltrated by tumor cells (Fig. 2). Atypical mitoses were rarely observed and necrosis was absent. Perineural and lymphovascular invasion were clearly observed (Fig. 3). These microscopic findings were consistent with the diagnosis of mesenteric paraganglioma.

Immunohistochemistry confirmed our diagnosis, as the tumor cells were positive for Synaptophysin and Chromogranin A, while S100 and glial fibrillary acidic protein (GFAP) markers emphasized the presence of sustentacular cells. Antigen Ki-67 (Ki-67) index was under 1\% (Fig. 4). Extra markers were ordered for differential diagnosis, namely pan cytokeratin (AE1/AE3), Desmin, clusted of differentiation 117 (CD117) and melanoma marker antibody (HMB45).

Finally, the histological grade of the tumor was correlated with the risk of malignancy using the adrenal pheochromocytoma and paraganglioma (GAPP) score. The score was calculated using different parameters such as histological pattern, score 1 (large and irregular cell nests); cellularity, score 2 (there were more than 250 cells/ $\mathrm{U}^{*}$ ) [*U = cells in unit

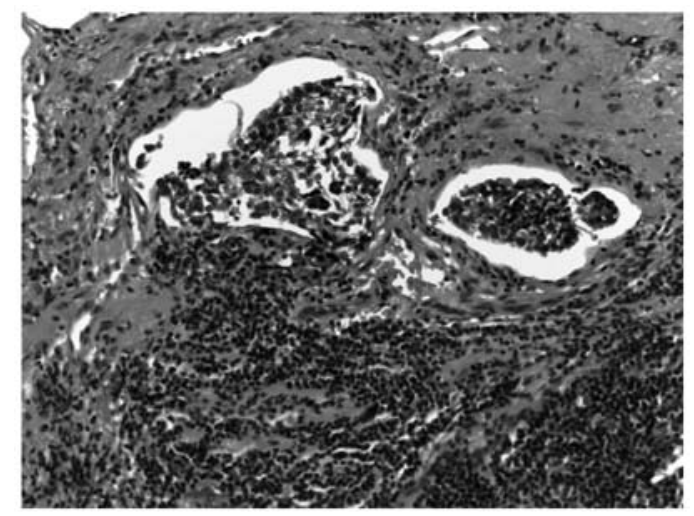

Figure 3. Vascular invasion with lymphovascular emboli (hematoxylin and eosin, $\mathrm{x} 20$ ).

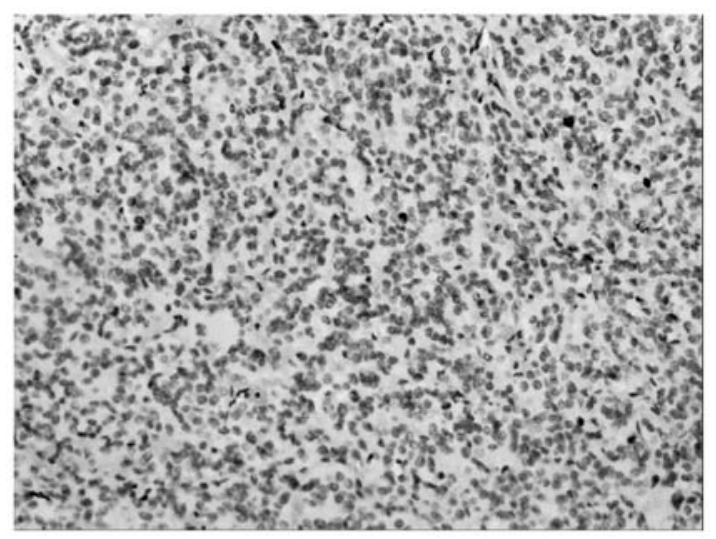

Figure 4. Proliferation index Ki-67 was under 1\% (Ki-67, x20).

of 10x10 $\mu \mathrm{m}$ under high power field (x400)]; comedo necrosis, score 0 (the necrosis was absent); vascular or capsular invasion, score 1 (both were present); Ki-67 labelling index, score $0(\mathrm{Ki}-67<1 \%)$; catecholamine type, score 0 (there was no documented production of catecholamines).

According to a total GAPP score of 4 , the paraganglioma was classified as moderately differentiated which corresponds to an intermediate metastasizing risk.

\section{Discussion}

The mesentery is a rare location for extra-adrenal paragangliomas. They are distributed along the para-aortic and paravertebral axis corresponding to the sympathetic nervous system. Abdominal occurrence is the best documented in the area corresponding to the organ of Zuckerkandl $(3,4)$.

The pathogenesis is not fully understood at the moment, but as much as $40 \%$ of these tumors have been associated with a germline mutation and therefore all patients with paragangliomas should be referred for clinical genetic testing. Hereditary cases may be associated with multiple endocrine neoplasia type 2, von Hippel-Lindau disease, familial paraganglioma, Carney triad and neurofibromatosis type 1 (5). According to the European Clinical Guideline, all patients diagnosed with pheochromocytoma and paraganglioma should be followed-up for at least 10 years or should be offered lifetime annual follow-up if criteria of high-risk were present (young age, 
hereditary disease, large tumor and the presence of a paraganglioma) (6). The clinical presentation of paraganglioma resembles symptoms of carcinoid syndrome, secondary to production of catecholamines. These symptoms may include sweating, diarrhea, anxiety and hypertension. However, it is not uncommon for a paraganglioma to present itself without any symptoms, as it was shown in our case.

Based on the 4th edition of WHO Endocrine Tumour Classification (2017), all pheochromocytomas and paragangliomas have a metastatic potential and they are all now considered malignant, as opposed to a previous division into malignant and benign tumors (7). However, the risk of metastasis is correlated to the risk stratification based on GAPP score which predicts the patient survival. GAPP score analyses six parameters (histological pattern, cellularity, comedo necrosis, vascular or capsular invasion, Ki-67 labelling index and catecholamine type) and evaluates the malignant grade of pheochromocytomas and paragangliomas. According to the total score, the tumor falls into one of three categories (poorly, moderately and highly differentiated) which are correlated to survival and metastatic rate (8). In our case, a score of 4 corresponds to a $60 \%$ metastatic rate and a $66.8 \% 5$-year survival (8).

Paragangliomas are microscopically described as solid nests of round and oval cells with abundant granular cytoplasm, which is either basophilic or acidophilic. These nests of cells are designated as 'Zellballen' pattern which is highly specific to paragangliomas. The nests are specifically surrounded by sustentacular cells showing dendritic features which are difficult to spot on classical hematoxylin-eosin stain, but can be highlighted with S100 marker (1). Melanin-like pigment might occur, in which case they are called 'Pigmented paragangliomas'. The stroma presents a prominent vascular network. In most cases the necrosis is absent and no rosettes and acini are present. Unusual mitoses are rare.

Pathological diagnosis of paragangliomas is often difficult to establish, especially in less common locations such as mesentery or when there is a lack of symptoms secondary to catecholamine excess. These tumors have a low incidence and immunohistochemistry is a compulsory tool for a correct differential diagnosis. The most important markers required for this diagnosis are chromogranin A (or dopamine $\beta$-hydroxylase) and S100. Chromogranin A is a specific neuroendocrine marker which is positive in all functioning and non-functioning paragangliomas, but, if negative, a diagnosis of paraganglioma should be excluded. Similarly, dopamine $\beta$-hydroxylase marker is also decisive for this diagnosis. S100 yields evidence for sustentacular cells. Other neuroendocrine markers such as CD56 and synaptophysin are also useful but not exclusive (8). Ki-67 proliferation index is required for the calculation of GAPP score.

Due to a lack of specific histological features such as zellballen pattern, we carefully approached the process of differential diagnosis. We considered several entities that could mimic this tumor. Firstly, reaction to keratin stains was analysed. Only a few rare paragangliomas such as gangliocytic and cauda equine-types were described in the literature as being positive to keratin stains and negativity to these markers is highly suggestive for paraganglioma (9). Therefore, AE1/AE3 marker was performed and a negative result excluded a neuroendocrine tumor, as all neuroendocrine tumors are usually positive to this stain. Similarly, negative reaction for CD117 and desmin ruled out a possible gastrointestinal stromal tumor. Metastasis of melanoma was also ruled out by a negative response for HMB45.

In conclusion, non-functioning paragangliomas situated in unusual locations such as the mesentery are challenging. In case of scarce specific features on classic stains, a careful approach in differential diagnosis should be considered. The critical markers for paragangliomas, chromogranin A and S100, should be used first in the process of diagnosis, followed by other valuable immunohistochemical markers to rule out other entities. A long-term follow-up is extremely important following the diagnosis of paraganglioma as all these tumors present malignant potential.

\section{Acknowledgements}

Not applicable.

\section{Funding}

Not funding was received.

\section{Availability of data and materials}

The datasets used and analyzed during the current study are available from the corresponding author on reasonable request.

\section{Authors's contributions}

AI performed the tumor histological examination and was a major contributor in the writing of the manuscript. IAU performed the immunohistochemistry examination of the tumor and was a major contributor in the writing of the manuscript. AP analyzed and interpreted the patient data regarding the hereditary involvement. MMC analyzed and interpreted the patient data regarding the neuroendocrine disease. RCP performed the radiography interpretation of the tumor and was a major contributor in the writing of the manuscript. FS analyzed and interpreted the genetic substrate of the disease. TC analyzed and interpreted the patient data regarding the surgery approach and was a major contributor in the writing of the manuscript. MCD analyzed and interpreted the patient data regarding the differential diagnosis of the patient and was a major contributor in the writing of the manuscript. All authors read and approved the final version of the manuscript.

\section{Ethics approval and consent to participate}

Not applicable.

\section{Patient consent for publication}

The patient provided written informed consent for the patient information to be published.

\section{Competing interests}

Not applicable. 


\section{References}

1. Asa $S$, Ezzat $S$ and Mete O: The diagnosis and clinical significance of paragangliomas in unusual locations. J Clin Med 7: $280,2018$.

2. Kudoh A, Tokuhisa Y, Morita K, Hiraki S, Fukuda S, Eguchi N and Iwata T: Mesenteric paraganglioma: Report of a case. Surg Today 35: 594-597, 2005.

3. Jaffer S and Harpaz N: Mesenteric paraganglioma: A case report and review of the literature. Arch Pathol Lab Med 126: 362-364, 2002.

4. Lack EE, Cubilla AL, Woodruff JM and Lieberman PH: Extra-adrenal paragangliomas of the retroperitoneum: A clinicopathologic study of 12 tumors. Am J Surg Pathol 4: 109-120, 1980.

5. Fujita T, Kamiya K, Takahashi Y, Miyazaki S, Iino I, Kikuchi H, Hiramatsu Y, Ohta M, Baba S and Konno H: Mesenteric paraganglioma: Report of a case. World J Gastrointest Surg 5: 62-67, 2013 .
6. Plouin PF, Amar L, Dekkers OM, Fassnacht M, GimenezRoqueplo AP, Lenders JW, Lussey-Lepoutre C and Steichen O; Guideline Working Group: European Society of Endocrinology Clinical Practice Guideline for long-term follow-up of patients operated on for a phaeochromocytoma or a paraganglioma. Eur J Endocrinol 174: G1-G10, 2016.

7. Lloyd RV, Osamura YR, Kloppel G and Rosai J: WHO classification of tumours of endocrine organs. WHO Press 31: 1770-1786, 2017.

8. Kimura N, Takekoshi K and Naruse M: Risk stratification on pheochromocytoma and paraganglioma from laboratory and clinical medicine. J Clin Med 7: E242, 2018.

9. Singh S, Asa SL, Dey C, Kennecke H, Laidley D, Law C, Asmis T, Chan D, Ezzat S, Goodwin R, et al: Diagnosis and management of gastrointestinal neuroendocrine tumors: An evidence-based Canadian consensus. Cancer Treat Rev 47: 32-45, 2016. 